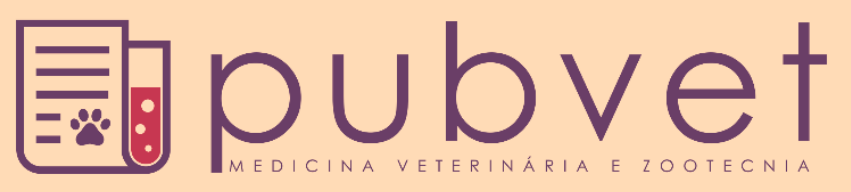

https://doi.org/10.31533/pubvet.v15n09a909.1-6

\title{
Luxação exposta da articulação tarso metatársica em potro: Relato de caso
}

\author{
Thales Rodrigues Costa $^{1} \bullet$, Jackson Brendo Gomes Dantas ${ }^{2} \bullet$ (D), Pedro Ferreira de Sousa \\ Júnior $^{2}{ }^{\circ}$, José Pires de Carvalho Neto ${ }^{3}$, Manuelle Rodrigues da Silva ${ }^{4}$, Larissa Maria \\ Feitosa Gonçalves $^{50}$, Antônio Augusto Nascimento Machado Júnior ${ }^{6}{ }^{\circ}$, Manoel Lopes da Silva Filho ${ }^{5 *} \bullet$ \\ ${ }^{I}$ Médico Veterinário do Município de Floriano, Piauí. Brasil. \\ ${ }^{2}$ Discente do Curso de Bacharelado em Medicina Veterinária da Universidade Federal do Piauí do Campus Professora Cinobelina Elvas, Bom Jesus, Piauí, Brasil. \\ ${ }^{3}$ Doutorando em Ciência Animal da Universidade Federal do Piauí do Campus Professora Cinobelina Elvas, Bom Jesus, Piauí, Brasil. \\ ${ }^{4}$ Docente da Faculdade de Tecnologia de Teresina-CET, Teresina, Piauí, Brasil. \\ ${ }^{5}$ Docente da Universidade Federal do Piauí do Campus Professora Cinobelina Elvas, Departamento de Reprodução Animal, Bom Jesus, Piauí, Brasil. \\ ${ }^{6}$ Docente da Universidade Federal do Piauí do Campus Ministro Petrônio Portela, Teresina, Piauí, Brasil. \\ *Autor para correspondência, E-mail: manoellopes@ufpi.edu.br
}

Resumo. As principais causas do afastamento dos cavalos atletas das atividades esportivas estão relacionadas com injurias que afetam o sistema locomotor, fato esse que, lesões locomotoras afastam mais animais do esporte quando comparado a alterações gástricas ou respiratórias. E nesse aparelho todas as estruturas como: ossos, tendões, articulações, estão sujeitas a sofrerem algum tipo de lesão, podendo acarretar em uma claudicação que por sua vez pode estar associada a diversos fatores como disfunções metabólicas, processos infecciosos, traumas externos ou alteração genética. Dentre as lesões locomotoras, as luxações são comuns e deve-se ter cuidado já que apresentam grande potencial degenerativo para a articulação. O presente trabalho tem como objetivo relatar um caso de um potro macho, mestiço, de aproximadamente quinze dias de vida com suspeita de luxação, onde foi confirmado através de exames de imagem o diagnóstico definitivo de luxação da articulação tarso metatársica. No entanto, mesmo com o tratamento de suporte com antibióticos, anti-inflamatórios, limpeza da lesão e bandagem, o animal continuou a definhar e perder peso por não conseguir se alimentar devido à evolução da septicemia, o que acarretou a um quadro de artrite séptica degenerativa o que levou a equipe médica optar após autorização do proprietário pela eutanásia do animal. Portanto o atendimento e diagnóstico precoce são de grande importância para o sucesso do tratamento evitando assim complicações posteriores e possível morte do animal.

Palavras chave: Emergência, locomotor, neonatologia

\section{Exposed luxation of tarsometatarsal articulation in the foal: case report}

Abstract. The main causes of the withdrawal of athletic horses from sports activities are related to injuries that affect the locomotor system, a fact that locomotor injuries keep more animals from sport when compared to gastric or respiratory changes. And in this device, all structures, such as bones, tendons, joints, are subject to suffering some type of injury, which can result in lameness, which in turn can be associated with several factors such as metabolic dysfunctions, infectious processes, external traumas or genetic alteration. Among locomotor injuries, dislocations are common and care must be taken since they have great degenerative potential for the joint. The present work aims to report a case of a male, mestizo foal, approximately fifteen days old with suspicion of dislocation, where the definitive diagnosis of dislocation of the metatarsal tarsus joint was confirmed through imaging exams. However, even with the supportive treatment with antibiotics, antiinflammatories, cleaning of the lesion and bandaging, the animal continued to wither and lose weight due to not being able to feed due to the evolution of septicemia, which resulted 
in septic arthritis. degenerative which led the medical team to opt after authorization from the owner for the euthanasia of the animal. Therefore, early care and diagnosis are of great importance for the success of the treatment, thus avoiding further complications and possible death of the animal.

Key words: Emergency, locomotor, neonatology

\section{Luxación expuesta de la articulación tarsometatarsiana en un potro: Reporte de caso}

Resumen. Las principales causas de la retirada de los caballos deportistas de las actividades deportivas están relacionadas con lesiones que afectan al aparato locomotor, hecho que las lesiones locomotoras alejan a más animales del deporte en comparación con los cambios gástricos o respiratorios. Y en este sistema, todas las estructuras, como huesos, tendones, articulaciones, están sujetas a sufrir algún tipo de lesión, que puede resultar en cojera, que a su vez puede estar asociada a varios factores como disfunciones metabólicas, procesos infecciosos, traumatismos externos. o alteración genética. Entre las lesiones del aparato locomotor, las luxaciones son comunes y se debe tener cuidado ya que tienen un gran potencial degenerativo para la articulación. El presente estudio tiene como objetivo reportar el caso de un potro macho mestizo de aproximadamente quince días de edad con sospecha de luxación, donde se confirmó el diagnóstico definitivo de luxación de la articulación del tarso metatarsiano mediante exámenes de imagen. Sin embargo, incluso con el tratamiento de apoyo con antibióticos, antiinflamatorios, limpieza de la lesión y vendaje, el animal siguió marchitándose y perdiendo peso por no poder alimentarse por la evolución de la septicemia, que derivó en artritis séptica degenerativa. Lo que llevó al equipo médico a optar previa autorización del propietario por la eutanasia del animal. Por tanto, la atención y el diagnóstico precoces son de gran importancia para el éxito del tratamiento, evitando así mayores complicaciones y la posible muerte del animal.

Palabras clave: Emergencia, locomotor, neonatología

\section{Introdução}

As lesões do sistema locomotor são o maior motivo de afastamento de cavalos das pistas, superando enfermidades respiratórias ou digestórias (Stashak, 2011; Wrigley, 2002). Dentre as causas de claudicação, diversas afetam as articulações, como: osteocondrose, osteocondrite dissecante (OCD), fraturas articulares, sinovite, capsulites, tendinites, desmites, luxação, rompimento de meniscos etc. (Cauvin et al., 1997).

Deve-se considerar que as claudicações correspondem a $67 \%$ dos acometimentos nos equinos atletas. Destas, cerca de $75 \%$ estão relacionadas a membros torácicos e $90 \%$ localizada distalmente ao carpo. As doenças ou fraturas do osso sesamóide distal ou navicular, correspondem a um terço das claudicações crônicas em membros torácicos e, $80 \%$ das claudicações distais ao carpo, estando correlacionada direta ou indiretamente com o pé (Escodro et al., 2007).

A origem da claudicação e dos problemas locomotores é multifatorial e pode estar relacionada com alterações em outros órgãos ou sistemas (Schwartz \& Madson, 2014). Podem ser consequência de processos infecciosos ou metabólicos, assim como ter origem genética, nutricional, no manejo ou ambiente. Acredita-se que as perdas relacionadas com a claudicação, incluindo morte e descarte de animais, representam pelo menos 1 a 5\% da produção (Schwartz \& Madson, 2014; Stashak, 2011).

As luxações são produzidas basicamente pelos mesmos mecanismos das entorses, só que de intensidade muito maior, suficiente para provocar perda total da relação das faces articulares, ruptura de ligamentos e às vezes, até desgarro da cápsula com extravasamento de líquido sinovial (Thomassian, 2005).

O exame radiográfico é a técnica de imagem mais utilizada para o diagnóstico de afecções articulares, no entanto trata-se de um método pouco sensível. A cartilagem articular não pode ser visualizada radiograficamente, exceto quando existe uma marcada diminuição do espaço articular e múltiplas 
imagens são necessárias para conseguir avaliar a estrutura articular completa (Mcllwraith, 2010). Os achados radiológicos tendem a refletir processos e lesões que ocorreram anteriormente ao longo do processo patológico, mas não mostram os processos que estão a ocorrer no momento (Caron, 2011). Ribeiro (2006) evidenciou pelas avaliações radiográficas articulares em 120 equinos, alta incidência de lesões em animais clinicamente normais ou que aparentavam acometimento leve. Afirmando que, a avaliação clínica do equino relacionada à sua utilização é preponderante em relação ao estudo radiográfico. O prognóstico do equino, depende do tratamento que o animal for submetido. Quanto mais cedo o tratamento for iniciado, melhor será a possibilidade de o animal voltar a sua atividade (Higgins \& Snyder, 2013).

Portanto, o presente trabalho tem como objetivo relatar um caso de luxação exposta da articulação tarsometatársica em potro e toda conduta realizada no caso em questão.

\section{Relato de caso}

Um potro macho, mestiço, de aproximadamente 15 dias de vida, foi atendido no Hospital Veterinário de Patos, Paraíba, com a queixa principal que o animal estava há dois dias sem tocar o membro pélvico direito no chão. $\mathrm{Na}$ anamnese o proprietário relatou que medicou o animal com anti-inflamatório, que o osso da articulação estava para fora da pele, está claudicando e não se alimentava direito. Ao exame físico, foi possível observar um edema no membro pélvico direito, presença de dor local, uma abertura na pele com um pouco de secreção, além da claudicação e dificuldade de locomoção. As mucosas estavam normais, sem desidratação aparente, animal apresentava-se em estação, com movimentos intestinais normais, tempo de perfusão capilar $\leq 2 \mathrm{~s}$, comportamento ativo, estado nutricional bom e temperatura de $38,8^{\circ} \mathrm{C}$. Após anamnese e exame físico, suspeitou-se de uma luxação exposta entre o tarso e o metatarso. Para o diagnóstico definitivo, o animal foi encaminhado para exame radiográfico, chegando ao diagnóstico definitivo de luxação da articulação tarso metatársica (Figura 1).

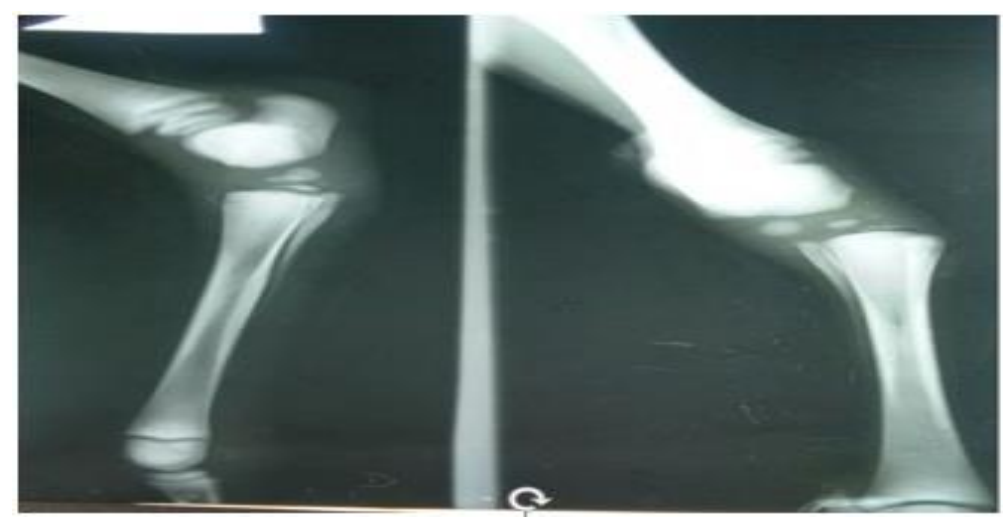

Figura 1. Radiografia do membro posterior direito, demonstrando o deslocamento entre os ossos do tarso e metatarso.

Após os exames, foi realizado limpeza e imobilização do membro, com uma bandagem fixa de material resistente e acolchoado, para que impedisse a entrada de sujidades e preservasse o ferimento e articulação sempre limpos, protegendo-o de maiores contaminações.

Para o tratamento, foi prescrito Cefalosporina 3mg/kg SID, Pentabiótico ${ }^{\circledR} 2,5 \mathrm{~mL}$, IM de $48 / 48$ horas, para evitar contaminação na articulação, Meloxicam $3 \% 1 \mathrm{~mL}$, IM para alívio da dor e compressa gelada duas vezes ao dia para diminuir o edema local. Em seguida o potro foi levado para uma baia com piso de areia junto com a sua mãe. No dia seguinte, observou-se que o animal passava a maior parte do tempo deitado e se levantava com dificuldades, na maioria das vezes para mamar. No segundo e terceiro dia, o animal apresentava-se com quadro inalterado em relação ao primeiro dia. Ainda no terceiro dia, foi realizada a troca de bandagem, limpeza com solução fisiológica e aplicação de alantoina local. Com cinco dias de internado observou-se fraqueza do animal por não estar conseguindo levantar e se alimentar, suplementando-o então com leite de vaca. No oitavo dia, retiraram a bandagem para avaliação e limpeza do ferimento. Observando que a articulação do animal estava sendo comprometida e o estado nutricional cada vez pior, a equipe cirúrgica interviu para avalia-lo. 


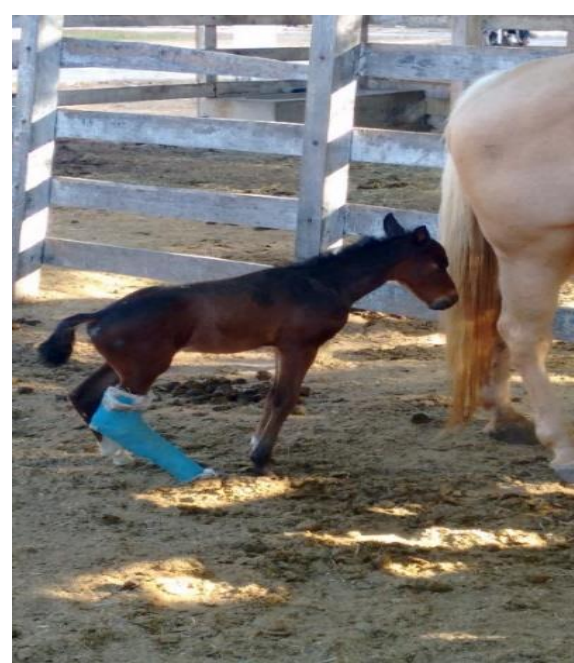

Figura 2. Animal com o membro imobilizado

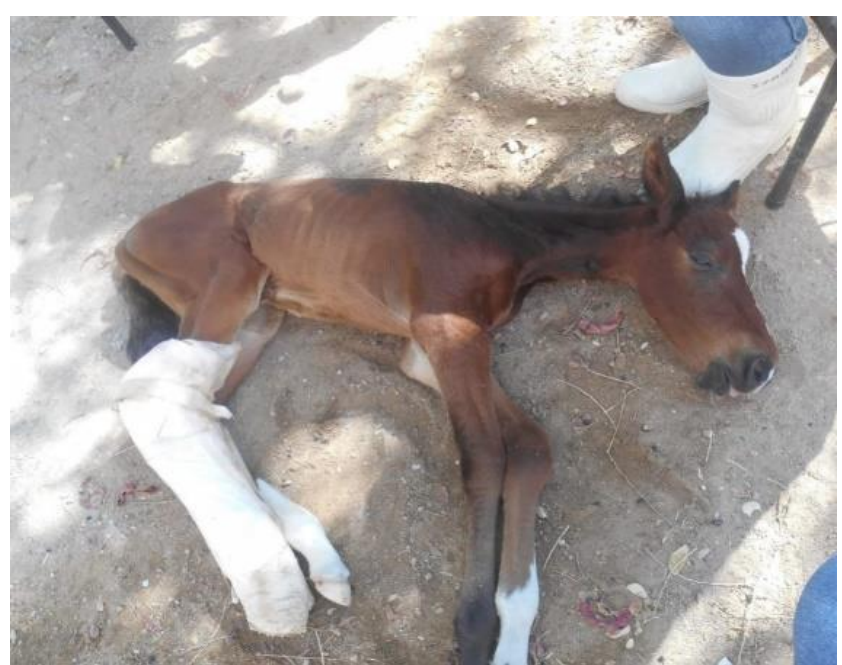

Figura 3. Potro em decúbito lateral com quadro nutricional debilitado.

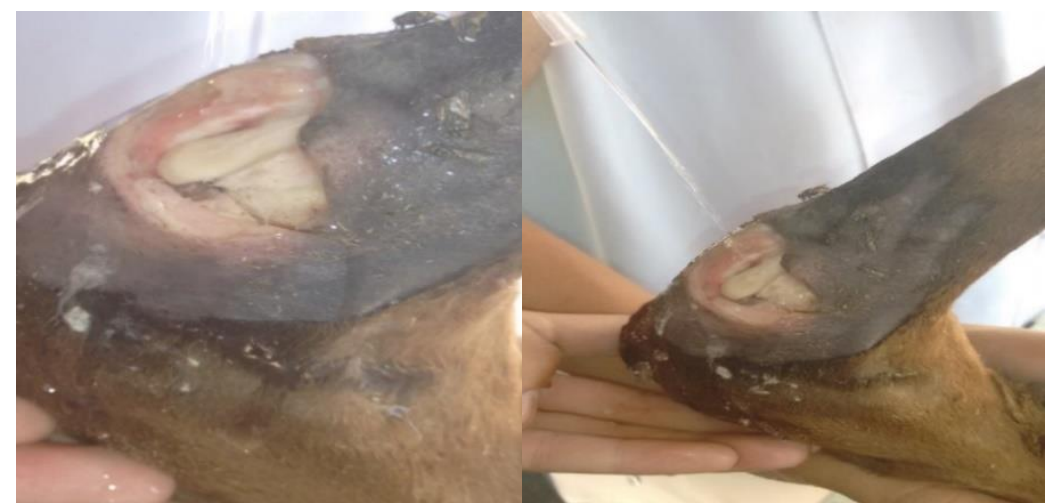

Figura 4. Articulação comprometida com artrite séptica.

Devido ao estado nutricional e comprometimento da articulação por uma artrite séptica, a equipe optou por eutanasiá-lo. Na necropsia, foi observada artrite séptica na articulação tarso metatársica.

\section{Discussão}

Com os dados obtidos pelo exame clínico executado, assim como a anamnese, pode-se chegar a um diagnóstico presuntivo mais rápido e realizar uma terapia correta, o que de acordo com Stashak (2011) durante a realização da anamnese, deve-se colher informações sobre o tempo e a intensidade da claudicação, os sinais e a atividade imediatamente precedente à instalação do processo, assim como quais quer terapias e tratamentos prévios realizados e se os resultados foram positivos. No entanto Ireland et al. (2012) relatam que a prevalência estimada de doenças por informações dos proprietários é consideravelmente menor quando comparadas pelo médico veterinário.

Para o diagnóstico definitivo, realizaram-se radiografias do membro, que para Feitosa (2014) é necessário para o complemento do diagnóstico, e para que seja confiável é preciso conhecimento da técnica e dos equipamentos utilizados, bem como ter a capacidade de interpretação dos resultados obtidos. Speirs (1999) relata que as posições são de fundamental importância, onde para o tarso, as posições são a lateromedial, a dorsoplantar, as oblíquas $35^{\circ}$ dorsolateral-plantaromedial e dorsomedial-plantarolateral.

A utilização da imobilização do membro do animal foi de fundamental importância mesmo o animal tendo sofrido uma luxação exposta, pois De Marval et al. (2004) descrevem que as resoluções de luxação fechada se dão por meio da redução e imobilização com gesso. No entanto, as luxações expostas, quase que invariavelmente evoluem para uma artrite séptica, necessitando de terapia intravenosa local e sistêmica, além de imobilização. A estabilidade pode ser recuperada por imobilização com gesso ou pela realização de artrodese. 
Em relação a artrite séptica equina (ASE) Botejo et al. (2012) relata que é uma patologia que acomete os potros bem como equinos adultos, não tendo predisposição quanto à idade, sexo ou raça. É o problema mais grave observado nas articulações dos equinos segundo (Thomassian, 2005), sendo uma doença progressiva e erosiva das articulações, que leva o animal a uma claudicação severa e requer um tratamento intenso e prolongado (Stashak, 2011). Em neonatos, o risco de infecção articular é maior durante os primeiros 30 dias de vida devido a parcial ou completa falha na transferência passiva de imunoglobulinas (Thomassian, 2005), que pode desencadear um quadro de onfalite (infecção do umbigo), sendo a poliartrite, a infecção secundária mais frequente (Riet-Correa et al., 2007). Segundo Lugo \& Gaughan (2006) relatam que para uma compreensão da etiopatogenia de casos de artrite séptica é necessário um histórico clinico completo que indique fonte de infecção. Potros jovens desenvolvem artrite séptica após episódio de bacteremia, resultantes, principalmente de infecções pulmonares e umbilicais. Para Jubb et al. (2007) algumas bactérias como Actinobacillus equuli, Escherichia coli, Klebsiella spp., Rhodococcus equi, Salmonella spp., Streptococcus (grupo C) spp podem estar envolvidas.

No que diz respeito ao tratamento realizado para combate da artrite séptica Stashak (2011) afirma que o tratamento tem como objetivo eliminar o microrganismo causador da doença, remover os produtos deletérios da inflamação sinovial e a fibrina que podem danificar a cartilagem articular. Para evitar lesões crônicas, o tratamento deve ser iniciado o mais cedo possível. Sendo assim, agentes antimicrobianos, incluindo sulfas, tetraciclinas ou penicilina-estreptomicina, podem ser utilizados (RietCorrea et al., 2007), tanto por via sistêmica quanto intra-articular. Também pode ser realizada drenagem e lavagem da articulação (Smith, 2006). Características desejáveis do antimicrobiano incluem boa susceptibilidade comum de agentes infecciosos, a atividade bactericida, a capacidade de atingir níveis terapêuticos nos tecidos sinoviais e ossos, boa potência, toxicidade mínima, efeitos colaterais mínimos ao paciente, e acessibilidade (Bertone, 1996).

Antibióticos potentes, de amplo espectro de regimes devem ser usados antes que os resultados da cultura estejam disponíveis. Antibioticoterapia sistêmica é sempre praticada com penicilina/gentamicina em combinação, cefalosporina, gentamicina e trimetropimsulfa em combinações são as mais comumente usadas (Stashak, 2011). O uso de agentes anti-inflamatórios não-esteroidais, como a fenilbutazona, pode ser apropriado, mas deve-se ter muito cuidado na avaliação do progresso do tratamento quando o animal recebe a fenilbutazona. Os AINEs podem mascarar muito efetivamente a dor associada à artrite infecciosa (Smith, 2006).

Para potros o prognóstico é inferior à dos adultos, devido a complicações associadas com envolvimento de múltiplos órgãos e septicemia. Algumas infecções mistas são difíceis de serem eliminadas, e com o tempo, o osso pode ser penetrado causando uma osteomielite, como pode também desenvolver danos irreversíveis à cartilagem articular causando, uma osteoartrite (Auer \& Stick, 2018). O prognóstico em longo prazo pode ser muito bom se a infecção for controlada cedo. Vários meses de repouso devem ser concedidos após qualquer episódio de artrite séptica para permitir que a cartilagem articular possa recuperar a forma normal de matriz extracelular e sua função. Exercícios devem ser aplicados mesmo se a condição parecer ter sido resolvida rapidamente, exames radiográficos devem ser realizados à retomada do exercício, onde são indicados para avaliar se as patogenias de longo prazo não resultaram em efeitos negativos (Higgins \& Snyder, 2013).

\section{Conclusão}

Neste estudo concluímos a importância de se chegar a um diagnóstico presuntivo mais rápido e realizar uma terapia com base na anamnese, exame clínico, bem como exames complementares, para que se possa evitar complicações posteriores, neste caso uma luxação exposta evoluiu a uma artrite séptica, uma patologia progressiva e erosiva das articulações, que leva o animal a uma claudicação severa e irreversível o que levou a se decidir pela a eutanásia.

\section{Referências bibliográficas}

Auer, J. A., \& Stick, J. A. (2018). Equine surgery-E-Book. Elsevier Health Sciences.

Bertone, A. (1996). Infectious arthritis. In M. C. I. L. Wrath \& G. Trotter (Eds.), Joint disease in the horse (pp. 397-409). Elselvier Saunders. 
Botejo, C.S.; Lima, J.S.; Souza, A.A.F.; Souza, F.S.; Bayeux, J.J. Artrite séptica equina em neonato decorrente de onfaloflebite diagnosticada na cidade de Manaus-AM. http://jjvet.wordpress.com/2012/04/26/artrite-septica-equina-em-neonato-decorrente-deonfaloflebite-diagnosticada-na-cidade-de-manaus-am/ Publicado: 26 de abril de 2012.

Caron, J. P. (2011). Osteoarthritis. In N. W. Ross \& S. J. Dyson (Eds.), Diagnosis and management of lameness in the horse (pp. 655-668). Elselvier Saunders.

Cauvin, E. R. J., Munroe, G. A., Boswell, J., \& Boyd, J. S. (1997). Gross and ultrasonographic anatomy of the carpal flexor tendon sheath in horses. Veterinary Record, 141(19), 489-495. https://doi.org/10.1136/vr.141.19.489.

De Marval, C. A., Gheller, V. A. I., Alves, G. E. S., Leal, B. B., \& Borges, K. D. S. (2004). Artrodese da articulação metacarpofalangeana para tratamento de luxação exposta em eqüino. Brazilian Journal of Veterinary Research and Animal Science, 41, 248. https://doi.org/10.11606/issn.2318-3659.v41isupl.p248-248.

Escodro, P. B., Gianini, C., \& Lopes, P. F. R. (2007). Considerações sobre as fraturas dos ossos metacárpicos (metatársicos) acessórios nas desmites do suspensório do boleto. Revista Brasileira de Medicina Veterinária Equina, 2(13), 24-27.

Feitosa, F. L. F. (2014). Semiologia veterinária: A arte do diagnóstico. Grupo Gen-Editora Roca Ltda. Higgins, A. J., \& Snyder, J. R. (2013). The equine manual E-Book. Elsevier Health Sciences.

Ireland, J. L., Clegg, P. D., McGowan, C. M., McKane, S. A., Chandler, K. J., \& Pinchbeck, G. L. (2012). Comparison of owner-reported health problems with veterinary assessment of geriatric horses in the United Kingdom. Equine Veterinary Journal, 44(1), 94-100. https://doi.org/10.1111/j.2042-3306.2010.00361.x.

Jubb, K. V. F., P.C., K., \& M.G., M. (2007). Pathology of domestic animals (Vol. 2). Academic press.

Lugo, J., \& Gaughan, E. M. (2006). Septic arthritis, tenosynovitis, and infections of hoof structures. Veterinary Clinics: Equine Practice, 22(2), 363-388. https://doi.org/10.1016/j.cveq.2006.03.005.

McIlwraith, C. W. (2010). Recent advances in diagnosis of equine joint disease. Proceedings of the 17th Kentucky Equine Research Nutrition Conference. Kentucky Equine Research, 23-33.

Riet-Correa, F., Méndez, F., Carmen Schild, M., Riet-Correa, A. L. F., Schild, A. L., \& Carmen, M. M. (2007). Doenças de ruminantes e eqüinos. Varela.

Ribeiro, M.G. Aspectos clínicos, cirúrgicos e radiológicos das principais fraturas ocorridas em equinos puro sangue inglês - Parte I e Parte II. 2006.

Schwartz, K., \& Madson, D. (2014). Sorting out joint disease and lameness. In National Hog Farmer (Vol. 1).

Smith, M. O. (2006). Tratado de medicina interna de grandes animais (Vol. 1). Manole.

Speirs, V. C. (1999). Exame clínico de eqüinos. Artmed.

Stashak, T. S. (2011). Claudicação em eqüinos segundo Adams. Editora Roca.

Thomassian, A. (2005). Enfermidades dos cavalos. Livraria Varela.

Wrigley, R. H. (2002). Ultrasonography of the tendons, ligaments, and joints. In T. S. Sthashak (Ed.), Adam's lameness in horses. Lippincott Williams \& Wilkins.

Histórico do artigo:

Recebido: 5 de maio de 2021

Aprovado: 10 de junho de 2021
Licenciamento: Este artigo é publicado na modalidade Acesso Aberto sob a licença Creative Commons Atribuição 4.0 (CC-BY 4.0), a qual permite uso irrestrito, distribuição, reprodução em qualquer meio, desde que o autor e a fonte sejam devidamente creditados. 